\title{
A Light Weight Rotary Double Pendulum: Maximizing the Domain of Attraction
}

\author{
R. W. Brockett* and Hongyi Li* \\ Engineering and Applied Sciences \\ Harvard University \\ Cambridge, MA 02138, USA \\ \{brockett, hongyi\}ehrl.harvard.edu
}

\begin{abstract}
The usual application of feedback stabilization is based on linearization and the assumption of linear controllability. Least squares optimal control is often used to determine a set of feedback gains that will stabilize the system. In addition to the commonly acknowledged challenges arising when this methodology is applied to open-loop unstable systems with significant nonlinearities, there are also significant problems which are not given prominence in the literature. These include the role of high gain feedback in exciting vibrations in unmodeled modes and the possibility of lightly damped oscillatory modes accentuating the effect of the nonlinearities. In this paper we report some experimental results relating to these points and suggest some techniques for dealing with them.
\end{abstract}

\section{INTRODUCTION}

This paper describes an experimental effort concerned with the stabilization of a rotary double link pendulum. This problem, and several related ones, has a long history going back several decades. In the literature the name of Furuta stands out because of his early interest in these problems and the steady stream of experimental results reported by his group [2], [5], [1]. Our approach differes from those previously reported not in terms of the basic form of the mechanics, but rather in terms of important aspects of the experimental apparatus. Most notably, we are working with a system with the following attributes:

1) All measurement signals are transmitted over a wireless link delivering 50 samples per second. This removes the need for wires connecting the moving parts to a fixed platform but introduces some latency in the data path and limits the sampling frequency.

2) The apparatus is light weight and has low inertia. This results in a structure with low stiffness and a tendency to vibrate.

3) The motor is a low torque, completely integrated amplifier/motor/controller unit rather than a high performance direct drive motor. This makes it necessary to design around the possibility of torque saturation.

In attempting to further develop the rotary link double pendulum stabilization technology, several challenges present themselves. The principal ones are.

1) The emergence of vibrational modes associated with unmodeled dynamics associated with the elasticity of

\footnotetext{
*This work was supported in part by the National Science Foundation under Yale prime CCR 9980058 and the US Army Research Office under Boston University prime GC169369 NGD, the National Science Foundation grant number EIA 0218411
}

the structure.

2) The problem of avoiding high feedback gains which can lead to torque saturation, noise amplification, and undesirable vibration.

3) A reduction in the size of the domain of asymptotic stability.

The general problem of stabilization via feedback has received considerable attention in both a linear and nonlinear context. Indeed, one of the central questions in classical control theory is the problem of avoiding instability in feedback systems. In this paper we are concerned with the problem of designing a control law that provides an adequate domain of attraction for a nonlinear problem of the form

$$
\dot{x}=f(x)+g(x) u
$$

under the hypothesis that $f(0)=0 ; g(0) \neq 0$. We assume that $f$ and $g$ are smooth functions and that the linearized system is controllable. Such questions are widely studied in the literature, being motivated by a variety of concrete problems including experimental work on stabilizing various types of open loop unstable mechanical systems.

Because we assume that the linearization of the system at $x=0$ is controllable, in theory there always exists a linear control law that stabilizes the null solution. Moreover, there exists a change of coordinates valid in a neighborhood of the origin that makes $g$ equal to a constant vector so we can carry out the first step in the feedback linearization procedure. A precise question along these lines can be formulated as follows.

Linear Feedback Stabilization Problem: Given a system of the form $\dot{x}=f(x)+G(x) u$ with $f(0)=0$ and $g(0) \neq 0$, find the linear time invariant control law $u=K x$ such that the null solution of $\dot{x}=f(x)+G(x) K x$ is asymptotically stable and the domain of attraction of 0 is as large as possible in the sense that it contains a ball of the form $\|x\| \leq a$ with $a$ maximal.

\section{A Hierarchy OF MEChanicAl Systems}

Our ultimate goal is to shed further light on the problem of enlarging the domain of attraction for nonlinear control systems. The platform that we use for the experiments to be described later consists of a horizontal link, driven by a servo motor, and two vertical links that moves freely in the plane perpendicular to the horizontal link, as shown in Fig. 4. Both this type of system and several other simpler mechanical 
systems have been extensively studied in the literature. In order to maximize the usefulness of this paper and to put our results in a broader context, we compare this system with a general version of the well known ball and beam system and the double pendulum on a cart system. We will see that these form a progression with the ball and beam being the least complex, the double pendulum being of intermediate complexity and the rotary pendulum being the most complex. For more about classical mechanics, see Whittaker [4].

\section{A. The Ball, Beam, Cart}

This is the least complicated system in our hierarchy. Consider the system ball and beam on a cart as shown in Fig. 1. The kinetic energy is

$$
\begin{gathered}
K=\frac{1}{2} m_{5}\left[\left(\dot{x}_{5}+\dot{x}_{1} \cos x_{3}\right)^{2}+\left(x_{5} \dot{x}_{3}-\dot{x}_{1} \sin x_{3}\right)^{2}\right] \\
+\frac{1}{2} I_{5}\left(\frac{\dot{x}_{5}}{r_{5}}-\dot{x}_{3}\right)^{2}+\frac{1}{2} m_{3} \dot{x}_{1}^{2} \\
+\frac{1}{2} I_{3} \dot{x}_{3}^{2}-m_{3} l_{3} \dot{x}_{1} \dot{x}_{3} \cos x_{3}
\end{gathered}
$$

Where $x_{5}$ is the position of the center of the ball relative to the joint of the beam. $r_{5}$ is the radius of the ball. $x_{3}$ is the angular position of the beam. $x_{1}$ is the position of the cart. All $x_{i}$ 's are on the same vertical plane. $m_{3}, m_{5}, I_{3}$, and $I_{5}$ are the mass and momentum of inertia of the ball and beam respectively. We assume to have direct control over the acceleration of the cart, i.e., $\ddot{x}_{1}=u$. The potential energy is

$$
V=m_{5} g x_{5} \sin x_{3}+m_{3} g l_{3} \cos x_{3} .
$$

The Euler-Lagrange equations take the form

$$
F(x) \ddot{x}+G(x, \dot{x})+V(x)=u b(x)
$$

with $x=\left[x_{1}, x_{3}, x_{5}\right]^{T}$

$$
\begin{gathered}
F(x)=\left[\begin{array}{ccc}
1 & 0 & 0 \\
0 & m_{5} x_{5}^{2}+I_{5}+I_{3} & -\frac{I_{5}}{r_{5}} \\
0 & -\frac{I_{5}}{r_{5}} & m_{5}+\frac{I_{5}}{r_{5}^{2}}
\end{array}\right] \\
G(x, \dot{x})=\left[\begin{array}{c}
0 \\
2 m_{5} x_{5} \dot{x}_{3} \dot{x}_{5} \\
-m_{5} x_{5} \dot{x}_{3}^{2}
\end{array}\right] \\
0 \\
0(x)=\left[\begin{array}{c}
-m_{3} l_{3} g \sin x_{3}+m_{5} g x_{5} \cos x_{3} \\
m_{5} g \sin x_{3}
\end{array}\right]
\end{gathered}
$$

and $b(x)$ is given by

$$
b(x)=\left[\begin{array}{c}
1 \\
m_{3} x_{5} \sin x_{3}+m_{3} l_{3} \cos x_{3} \\
-m_{5} \cos x_{3}
\end{array}\right]
$$

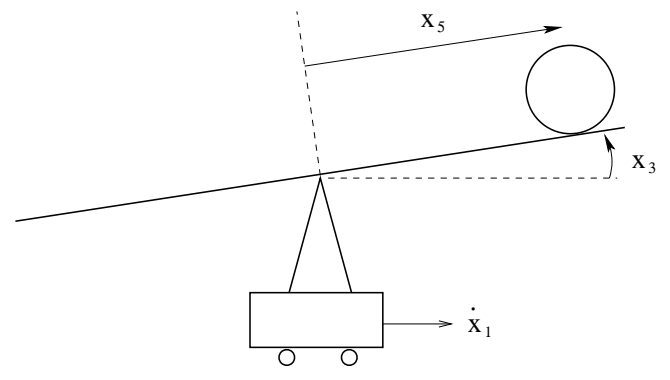

Fig. 1. Ball, Beam, Cart

Linearization of the Ball-Beam on Cart

$$
F \ddot{x}+V x=u b
$$

with

$$
\begin{gathered}
F=\left[\begin{array}{ccc}
1 & 0 & 0 \\
0 & I_{5}+I_{3} & -\frac{I_{5}}{r_{5}} \\
0 & -\frac{I_{5}}{r_{5}} & m_{5}+\frac{I_{5}}{r_{5}^{2}}
\end{array}\right] \\
V=\left[\begin{array}{ccc}
0 & 0 & 0 \\
0 & -m_{3} l_{3} g & m_{5} g \\
0 & m_{5} g & 0
\end{array}\right], \quad b=\left[\begin{array}{c}
1 \\
m_{3} l_{3} \\
-m_{5}
\end{array}\right]
\end{gathered}
$$

Transfer function $G(s)$ from $u$ to $x$ can be most easily obtained from the linearization in second order form,

$$
F s^{2} X(s)+V X(s)=U(s) b
$$

This yields the transfer function

$$
G(s)=\left[\begin{array}{c}
\frac{1}{s^{2}} \\
\frac{p_{22} s^{2}+p_{20}}{q_{4} s^{4}+q_{2} s^{2}+q_{0}} \\
\frac{p_{32} s^{2}+p_{30}}{q_{4} s^{4}+q_{2} s^{2}+q_{0}}
\end{array}\right]
$$

with

$$
\begin{aligned}
p_{22} & =-m_{5} r_{5} I_{5}+m_{3} l_{3}\left(m_{5} r_{5}^{2}+I_{5}\right) \\
p_{20} & =-m_{5}^{2} r_{5}^{2} g \\
p_{32} & =-m_{5} r_{5}^{2}\left(I_{3}+I_{5}\right)+m_{3} l_{3} I_{5} \\
p_{30} & =2 m_{3} m_{5} l_{3} r_{5} g \\
q_{4} & =m_{5} r_{5}^{2}\left(I_{3}+I_{5}\right)+I_{3} I_{5} \\
q_{2} & =2 m_{5} r_{5} I_{5} g-m_{3} l_{3} g\left(m_{5} r_{5}^{2}+I_{5}\right) \\
q_{0} & =-m_{5}^{2} r_{5}^{2} g
\end{aligned}
$$




\section{B. Cart with the double pendulum}

The coupled equations of motion that describe the dynamics of the pendulum, as shown in Fig. 2, are described by the Euler-Lagrangian equation

$$
F(\theta) \ddot{\theta}+G(\theta, \dot{\theta})+V(\theta)=u b(\theta)
$$

with $\theta=\left[\theta_{1}, \theta_{2}, \theta_{3}\right]^{T}$

$$
\begin{aligned}
& F(\theta)= \\
& {\left[\begin{array}{ccc}
1 & 0 & 0 \\
0 & I_{2}+m_{3} L_{2}^{2} & m_{3} l_{3} L_{2} \cos \left(\theta_{2}-\theta_{3}\right) \\
0 & m_{3} l_{3} L_{2} \cos \left(\theta_{2}-\theta_{3}\right) & I_{3}
\end{array}\right]} \\
& G(\theta, \dot{\theta})=\left[\begin{array}{c}
m_{3} l_{3} L_{2} \dot{\theta}_{3}^{2} \sin \left(\theta_{2}-\theta_{3}\right) \\
-m_{3} l_{3} L_{2} \dot{\theta}_{2}^{2} \sin \left(\theta_{2}-\theta_{3}\right)
\end{array}\right] \\
& V(\theta)=\left[\begin{array}{c}
0 \\
-\left(m_{2} g l_{2}+m_{3} g L_{2}\right) \sin \theta_{2} \\
-m_{3} g l_{3} \sin \theta_{3}
\end{array}\right]
\end{aligned}
$$

and $b(\theta)$ is given by

$$
b(\theta)=\left[\begin{array}{c}
1 \\
-\left(m_{2} l_{2}+m_{3} L_{2}\right) \cos \theta_{2} \\
-m_{3} l_{3} \cos \theta_{3}
\end{array}\right]
$$

Where $I_{2}$ and $I_{3}$ are the moments of inertia of link 2 and link 3 with respect to their joints $p_{2}$ and $p_{3}$ respectively. $L_{2}$ is the length of link 2 , while $l_{2}$ and $l_{3}$ are the distance from the center of mass to their joints of link 2 and link 3 respectively. In this problem, we can directly control the acceleration of the motor. Thus the motor equation is simply a double integrator $\ddot{\theta}_{1}=u$. Its linearization is identical to the rotary double pendulum as describe in the following subsection if the motor position $\theta_{1}$ is replaced by $L_{1} \theta_{1}$.

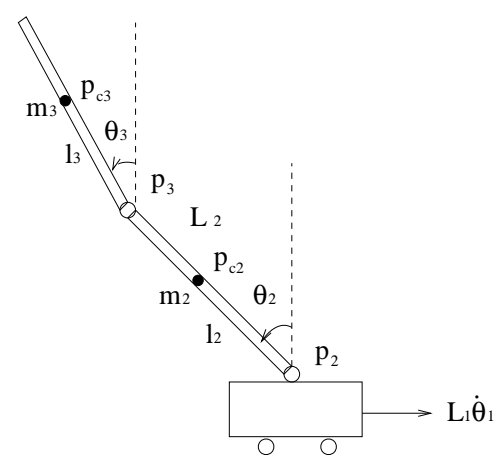

Fig. 2. The double pendulum on a cart

\section{Rotary Double Pendulum}

The coupled equations of motion that describe the dynamics of the pendulum, as shown in Fig. 3, are described by the Euler-Lagrangian equation

$$
F(\theta) \ddot{\theta}+G(\theta, \dot{\theta})+V(\theta)=u b(\theta)
$$

with $\theta=\left[\theta_{1}, \theta_{2}, \theta_{3}\right]^{T}$

$$
\begin{gathered}
F(\theta)=\left[\begin{array}{ccc}
1 & 0 & 0 \\
0 & I_{2}+m_{3} L_{2}^{2} & m_{3} l_{3} L_{2} \cos \left(\theta_{2}-\theta_{3}\right) \\
0 & m_{3} l_{3} L_{2} \cos \left(\theta_{2}-\theta_{3}\right) & I_{3}
\end{array}\right] \\
G(\theta, \dot{\theta})=\left[\begin{array}{c}
0 \\
g_{2}(\theta, \dot{\theta}) \\
g_{3}(\theta, \dot{\theta})
\end{array}\right] \\
V(\theta)=\left[\begin{array}{c}
-\left(m_{2} g l_{2}+m_{3} g L_{2}\right) \sin \theta_{2} \\
-m_{3} g l_{3} \sin \theta_{3}
\end{array}\right]
\end{gathered}
$$

and $b(\theta)$ is given by

$$
b(\theta)=\left[\begin{array}{c}
1 \\
-\left(m_{2} l_{2}+m_{3} L_{2}\right) L_{1} \cos \theta_{2} \\
-m_{3} l_{3} L_{1} \cos \theta_{3}
\end{array}\right]
$$

Where

$$
\begin{gathered}
g_{2}(\theta, \dot{\theta})=-\left[\frac{1}{2}\left(I_{2}+m_{3} L_{2}^{2}\right) \sin 2 \theta_{2}+m_{3} l_{3} L_{2} \cos \theta_{2}\right. \\
\left.\sin \theta_{3}\right] \dot{\theta}_{1}^{2}+m_{3} l_{3} L_{2} \dot{\theta}_{3}^{2} \sin \left(\theta_{2}-\theta_{3}\right) \\
g_{3}(\theta, \dot{\theta})=-\left(\frac{1}{2} I_{3} \sin 2 \theta_{3}+m_{3} l_{3} L_{2} \sin \theta_{2} \cos \theta_{3}\right) \dot{\theta}_{1}^{2} \\
-m_{3} l_{3} L_{2} \dot{\theta}_{2}^{2} \sin \left(\theta_{2}-\theta_{3}\right)
\end{gathered}
$$

$I_{2}$ and $I_{3}$ are the moments of inertia of link 2 and link 3 with respect to their joints $p_{2}$ and $p_{3}$ respectively. $L_{1}$ and $L_{2}$ are the length of link 1 and link 2 , while $l_{2}$ and $l_{3}$ are the distance from the center of mass to their joints of link 2 and link 3 respectively. In this problem, we can directly control the acceleration of the motor. Thus the motor equation is simply a double integrator $\ddot{\theta}_{1}=u$.

Associated with this nonlinear model is its linearization about $\theta_{i}=0, \dot{\theta}_{i}=0$ The corresponding terms are given by

$$
F \ddot{\theta}+V \theta=u b
$$

with

$$
\begin{gathered}
F=\left[\begin{array}{ccc}
1 & 0 & 0 \\
0 & I_{2}+m_{3} L_{2}^{2} & m_{3} l_{3} L_{2} \\
0 & m_{3} l_{3} L_{2} & I_{3}
\end{array}\right] \\
V=\left[\begin{array}{ccc}
0 & 0 & 0 \\
0 & -\left(m_{2} g l_{2}+m_{3} g L_{2}\right) & 0 \\
0 & 0 & -m_{3} g l_{3}
\end{array}\right]
\end{gathered}
$$




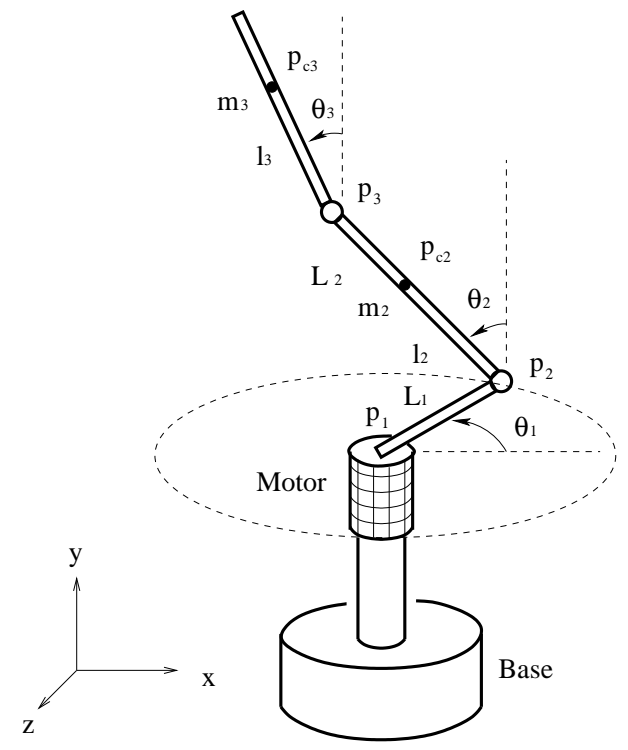

Fig. 3. The Rotary Double Pendulum

$$
b=\left[\begin{array}{c}
1 \\
-\left(m_{2} l_{2}+m_{3} L_{2}\right) L_{1} \\
-m_{3} l_{3} L_{1}
\end{array}\right]
$$

The transfer function from $u$ to $\theta$ is

$$
G(s)=\left[\begin{array}{c}
\frac{1}{s^{2}} \\
\frac{p_{22} s^{2}+p_{20}}{q_{4} s^{4}+q_{2} s^{2}+q_{0}} \\
\frac{p_{32} s^{2}+p_{30}}{q_{4} s^{4}+q_{2} s^{2}+q_{0}}
\end{array}\right]
$$

with

$$
\begin{aligned}
p_{22} & =-\left(m_{2} l_{2}+m_{3} L_{2}\right) I_{3} L_{1} \\
p_{20} & =\left(m_{2} l_{2} g+m_{3} L_{2} g+m_{3} l_{3} L_{2}\right) m_{3} l_{3} L_{1} \\
p_{32} & =-\left(I_{2}+m_{3} L_{2}^{2}\right)\left(m_{2} l_{2}+m_{3} L_{2}\right) L_{1} \\
p_{30} & =\left(m_{2} l_{2} g+m_{3} L_{2} g+m_{3} l_{3} L_{2}\right)\left(m_{2} l_{2}+m_{3} L_{2}\right) L_{1} \\
q_{4} & =\left(I_{2}+m_{3} L_{2}^{2}\right) I_{3} \\
q_{2} & =-\left[\left(I_{2}+m_{3} L_{2}^{2}\right) m_{3} l_{3}+\left(m_{2} l_{2}+m_{3} L_{2}\right) I_{3}\right] g \\
q_{0} & =\left(m_{2} l_{2} g^{2}+m_{3} L_{2} g^{2}-m_{3} l_{3} L_{2}^{2}\right) m_{3} l_{3}
\end{aligned}
$$

\section{Linearization IN A NEIGHBORHOOD}

If $f(0)=0$ then the linearization of

$$
\dot{x}=f(x)+g(x) u
$$

about $x=0$ to get

$$
\dot{\delta}=\left.\frac{\partial f}{\partial x}\right|_{0} \delta+g(0) u
$$

leads to theorems on local controllability and, via least squares theory, to feedback control laws resulting in asymptotic stability. However, in this case there are many stabilizing control laws and if the goal is to design a controller that will have a large domain of convergence, conventional linearization may not provide the best solution. Here we develop an alternative which we have found effective in controlling the rotary double pendulum.

Let $\Omega$ represent an open set in $\mathbb{R}^{n}$ that contains 0 . If we wish to find a matrix $A$ such that $A x$ approximates $f$ on the set $\Omega$ then we might choose $A$ so as to minimize

$$
\eta_{1}=\int_{\Omega}\|f(x)-A x\|^{2} d x
$$

Of course if $\Omega$ is very small the minimizing value of $A$ will be close to $\left.\frac{\partial f}{\partial x}\right|_{0}$ but if $\Omega$ is large it can be expected to be rather different. If we work with the Euclidean norm then the optimal value of $A$ is easily seen to be

$$
A^{*}=\int_{\Omega} f(x) x^{T} d x\left(\int_{\Omega} x x^{T} d x\right)^{-1}
$$

However, the possibility exists for improving the accuracy of this approximation through the use of feedback. Consider a refinement of the above procedure in which we select both $A$ and a feedback function $\alpha$ so as to minimize

$$
\eta=\int_{\Omega}\|f(x)-\alpha(x) g(x)-A x\|^{2} d x
$$

In this case we can see that for a given value of $A$ the best choice of $\alpha$ is the choice that cancels the error $f(x)-A x$ in the direction of $g$. This is given by

$$
\alpha(x)=\frac{\langle f(x)-A x, g(x)\rangle}{\langle g(x), g(x)\rangle}
$$

Thus the best value of $A$ minimizes

$$
\eta=\int_{\Omega}\left\|f(x)-\frac{\langle f(x)-A x, g(x)\rangle}{\langle g(x), g(x)\rangle} g(x)-A x\right\|^{2} d x
$$

Thus we can characterize $A$ implicitly by the linear equation in $A$,

$$
\begin{aligned}
& A^{*}\left(\int_{\Omega} x x^{T} d x\right) \\
& =\int_{\Omega}\left(f(x)-\frac{\left\langle f(x)-A^{*} x, g(x)\right\rangle}{\langle g(x), g(x)\rangle} g(x)\right) x^{T} d x
\end{aligned}
$$

Thus

$$
A^{*}\left(\int_{\Omega} x x^{T} d x\right)=M+\int_{\Omega}\left(\frac{\left\langle A^{*} x, g(x)\right\rangle}{\langle g(x), g(x)\rangle} g(x)\right) x^{T} d x
$$

This equation does not necessarily have a unique solution but the optimal value of $A x-\alpha(x) g(x)$ is unique.

For the rotary double pendulum it is possible to "regularize" the drift vector field $f$ through of a preliminary feedback as described here. In our experiments, this was an essential step in arriving at a stable system. 


\section{The High Gain Dilemma}

A major source of difficulty in achieving stability for the rotary double pendulum to be described below is the possibility of oscillations corresponding to unmodeled dynamics. For our system these are generally in the range of 6 to $8 \mathrm{~Hz}$. and are strongly destabilizing. It was observed experimentally that these oscillations could be avoided if the gains could be kept low. Sepulchre has several interesting examples on the high gain dilemma [3].

However, when stabilizing open loop unstable systems there are limitations on how low the gains can be. Letting $p(s)=\operatorname{det}(I s-A)$, the effect of the feedback term is to replace $p(s)$ by $p(s)+q(s)$. Suppose that $p(s)=$ $s^{n}+p_{n-1} s^{n-1}+\ldots+p_{0}$ has real coefficients and that we want to find the "smallest" polynomial $q(s)=q_{n-1} s^{n-1}+$ $q_{n-2} s^{n-2}+\ldots+q_{0}$ such that $p(s)+q(s)$ has all its roots in the left half-plane. Because the different entries in $q$ have different units we adopt a flexible characterization of size. involving a scaling vector $\alpha$. Consider

$$
|q|_{\alpha}=\sum \alpha_{i}\left|q_{i}\right|
$$

with with the components of $\alpha$ being positive numbers.

Recall that for a lossless mechanical systems, $p(D)$ is automatically an even function of $D$.

Fact 1: $p(s)$ is Hurwitz if and only if the zeros of $p_{e}(s)=$ $\left(p(s)+p(-s)\right.$ and the zeroes of $p_{o}(s)=p(s)-p(-s)$ are purely imaginary and interlace.

Fact 2: $p(s)$ is Hurwitz with all roots real and negative if and only $p\left(s^{2}\right)$ has all its roots on the imaginary axis. If this is the case, and if $p^{\prime}$ denotes the derivative of $p$ then $p\left(s^{2}\right)+p^{\prime}\left(s^{2}\right)$ is Hurwitz.

Example: Consider the polynomial $s^{2}\left(s^{4}-3 s^{2}+2\right)$. What polynomials can we add to this to get a polynomial that has all purely imaginary roots? Consider adding $a s^{4}+b s^{2}+c$. Conditions on the coefficients are

$$
\text { i) } a-3 \geq 0 \quad ; \quad(a-3)^{2}-4 b-8 \geq 0
$$

Possible solutions include $a=3, b=-2$ (minimal for $\alpha_{i}=1$ for all $i$ 's) and $b=-1, a=3+\sqrt{6}$, etc.

This analysis shows that there is a lower bound on the minimum gain required to stabilize an unstable mechanical system and that this can be characterized in terms of the determinantal polynomial.

\section{EXPERIMENTAL RESUlts}

The platform that we use for the experiments consists of a horizontal link, driven by a servo motor, and two vertical links that moves freely in the plane perpendicular to the horizontal link, as shown in Fig. 4. system thus provides us with enough bandwidth for controlling the pendulum. In order to measure the position of the vertical links, a rotary electric encoder is mounted on each of the joints. The encoder measures the absolute angular position (instead of incremental) and outputs analog signals. Then each analog

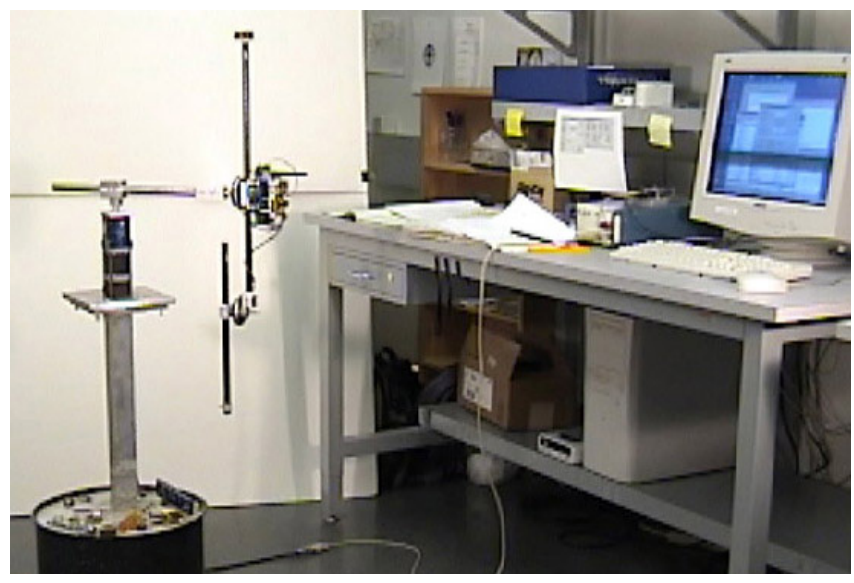

Fig. 4. The experimental setup

signal is converted into 12bits binary and then sent to the host computer through a RS232 radio link. The radio link can send 50 readings of both the encoders per second which limits the sample frequency of the whole system to $50 \mathrm{~Hz}$. Each encoder has accuracy of $.04 \mathrm{deg}$. The base of the pendulum is leveled with an error of $.06 \mathrm{deg}$.

We want to stabilize the pendulum at its inverse position. If we let $x=\left(\theta_{1}, \dot{\theta}_{1}, \theta_{2}, \dot{\theta}_{2}, \theta_{3}, \dot{\theta}_{3}\right)$, the linearized system equations become

$$
\dot{x}=A x+b u,
$$

The parameters we used in the double pendulum setup:

$$
\begin{aligned}
& g=9.81 ; \quad \text { gravitation constant } \\
& L_{1}=0.32 ; \quad L_{2}=0.264 ; \\
& m_{2} l_{2}=-0.0042 ; \quad m_{3} l_{3}=0.0023 ; \quad m_{3}=0.0795 ; \\
& I_{2}=0.0241 ; \quad I_{3}=7.5642 \times 10^{-4} .
\end{aligned}
$$

Thus at its inverse position, $x=0$,

$$
\begin{gathered}
A=\left[\begin{array}{cccccc}
0 & 1 & 0 & 0 & 0 & 0 \\
0 & 0 & 0 & 0 & 0 & 0 \\
0 & 0 & 0 & 1 & 0 & 0 \\
0 & 0 & 5.6491 & 0 & -0.6213 & 0 \\
0 & 0 & 0 & 0 & 0 & 1 \\
0 & 0 & -4.5347 & 0 & 30.3274 & 0
\end{array}\right] \\
b=\left[\begin{array}{c}
0 \\
1 \\
0 \\
-0.1640 \\
0 \\
-0.8414
\end{array}\right]
\end{gathered}
$$

The open loop transfer function is

$$
G(s)=C(s I-A)^{-1} b=\left[\begin{array}{c}
\frac{1}{s 2} \\
\frac{-0.1635 s 2+5.4862}{(s 2-5.536)(s 2-30.44)} \\
\frac{-0.8493 s 2+5.5392}{(s 2-5.536)(s 2-30.44)}
\end{array}\right] .
$$




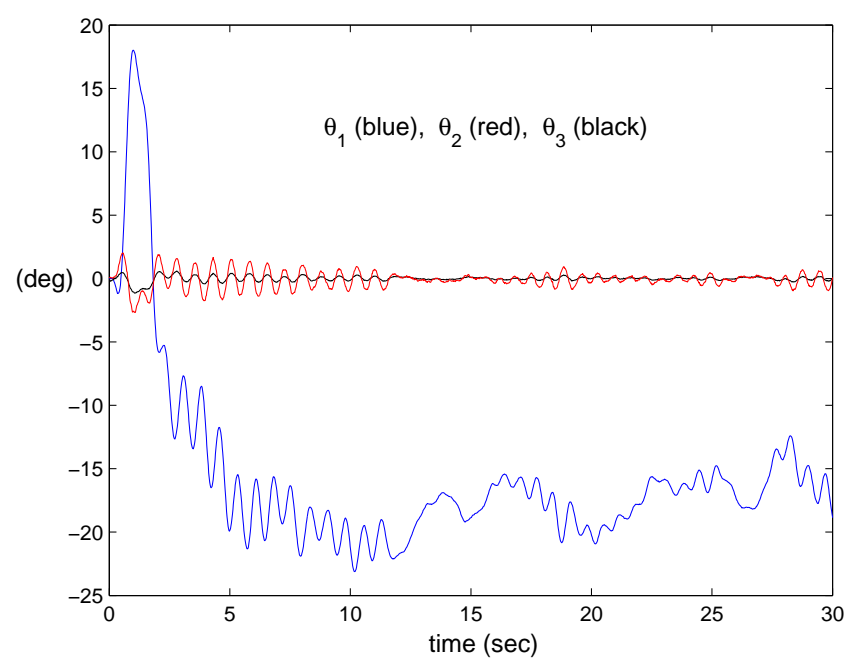

Fig. 5. Experimental data of up-up stabilizing control

The linear feedback gain we used after feedback linear approximation is

$$
\begin{aligned}
& k=\left[\begin{array}{llllll}
0.7 & 2.18 & 200 & 79.2 & -126 & -27.4
\end{array}\right] . \\
& e i g(A-b k)=\{-3.4682 \pm 0.5019 i,-2.2113 \text {, } \\
& -1.3882 \pm 2.3793 i,-0.5776\}
\end{aligned}
$$

as shown in Fig. 5.

\section{REFERENCES}

[1] K.J. Astrom, K. Furuta, M. Iwashiro and T. Hoshino. Energy based strategies for swinging up a double pendulum. Proceedings of 14th World Congress of IFAC 99. vol.14, pp283-8, 1999.

[2] K. Furuta, T. Okutani and H. Sone. Computer Control of a Double Inverted Pendulum. Computer and Electrical Engineering. vol.5, pp67-84, 1978.

[3] R. Sepulchre. Are basin of attraction easy to enlarge by feedback? preprint, 2003.

[4] E. T. Whittaker. A Treatise on the Analytical Dynamics of Particles and Rigid Bodies (4th ed). Cambridge University Press, Cambridge, U.K., 1937.

[5] M. Yamakita, K. Nonaka and K. Furuta. Swing up control of a double pendulum. Proceedings of 1993 American Control Conference. vol.3, pp2229-33, 1993. 